\title{
ILCEA
}

Revue de l'Institut des langues et cultures

d'Europe, Amérique, Afrique, Asie et Australie

45 | 2022

Femme(s) et sorcellerie en Espagne et en Italie à l'époque moderne

\section{La revanche de Circé : l'exemple de El Conde Partinuplés d'Ana Caro de Mallén}

La venganza de Circe: el ejemplo de El Conde Partinuplés de Ana Caro de Mallén

The Revenge of Circe: The Example of El Conde Partinuplés by Ana Caro de Mallén

Isabelle Rouane Soupault

\section{OpenEdition}

Journals

Édition électronique

URL : https://journals.openedition.org/ilcea/14739

DOI : 10.4000/ilcea.14739

ISSN : 2101-0609

Éditeur

UGA Éditions/Université Grenoble Alpes

Édition imprimée

ISBN : 978-2-37747-330-4

ISSN : 1639-6073

\section{Référence électronique}

Isabelle Rouane Soupault, "La revanche de Circé : l'exemple de El Conde Partinuplés d'Ana Caro

de Mallén », ILCEA [En ligne], 45 | 2022, mis en ligne le 31 janvier 2022, consulté le 31 janvier 2022

URL : http://journals.openedition.org/ilcea/14739 ; DOI : https://doi.org/10.4000/ilcea.14739

Ce document a été généré automatiquement le 31 janvier 2022.

(c) ILCEA 


\title{
La revanche de Circé : l'exemple de El Conde Partinuplés d'Ana Caro de Mallén
}

\author{
La venganza de Circe: el ejemplo de El Conde Partinuplés de Ana Caro de \\ Mallén \\ The Revenge of Circe: The Example of El Conde Partinuplés by Ana Caro de \\ Mallén
}

Isabelle Rouane Soupault

1 La figure archétypale de la sorcière hante l'imaginaire social et marque les consciences des cultures européennes depuis des temps immémoriaux. Les mystères qui l'entourent ont fortement contribué à sa marginalisation par des hommes soucieux de préserver leurs prérogatives. Le phénomène de polarisation contre la sorcière et ses avatars s'est aggravé à l'époque moderne dans les sociétés européennes tant cette femme incarnait en les combinant la puissance de la magie, la force de la spiritualité et une forme de connaissance scientifique dont la transmission était réservée à un petit nombre d'initiées. Nourrie par la tradition misogyne datant de l'Antiquité et par des peurs irrationnelles ravivées par l'actualité, la sorcière a été l'objet d'une répression particulièrement cruelle $\mathrm{au} \mathrm{xVII}^{\mathrm{e}}$ siècle. Les minutes des procès inquisitoriaux permettent d'élaborer un profil type pour cette accusée idéale (Jacques-Lefèvre, 2002). Les affirmations consignées dans ces textes témoignent de comportements mystérieux et fascinants qui effraient autant qu'ils attirent comme en rendent compte les célèbres ouvrages, le Malleus Maleficarum ou Marteau des sorcières de Henri Institoris et Jacques Spenger publié en 1486, et le Discours exécrable des sorciers de Henri Boguet publié en 1602. Parfois violente et cruelle, cette réaction antiféministe a pu justifier une mise à l'écart, présentée comme légitime, de toutes les femmes détentrices d'une forme de pouvoir. Ainsi, les écrivaines furent-elles réduites au silence, leurs créations furent le plus souvent occultées ou cantonnées dans des domaines approuvés par la bienséance (Gil-Albarellos \& Rodríguez Pequeño, 2006) : si la poésie pouvait apparaître comme une activité ornementale, au même titre que la pratique d'un instrument de musique, la 
publication de nouvelles ou, a fortiori, de pièces de théâtre, constituait une véritable transgression morale et sociale... Le petit nombre de pièces écrites par des femmes dramaturges dans l'Espagne du Xvir ${ }^{\mathrm{e}}$ siècle illustre la lente et chaotique émergence des autrices dans le domaine théâtral (Rouane Soupault, 2021). Le double seuil à franchir pour ces femmes, qui devaient parvenir non seulement à imposer la publication mais aussi organiser la représentation de leurs œuvres, les mettait le plus souvent en dehors du système existant.

2 L'exemple d'Ana Caro de Mallén fait donc figure d'exception remarquable dans ce contexte. Deux pièces sont signées de sa plume pour lesquelles elle adopte le modèlecadre de la comedia nueva initié par Lope de Vega: Valor, agravio y mujer (Le courage, l'outrage et la femme), une comédie de cape et d'épée, et El Conde Partinuplés (Le comte Partinuplés), d'inspiration à la fois mythologique et chevaleresque, où, précisément, la revendication de l'écriture théâtrale féminine s'exprime à travers la métaphore de la magie. L'écrivaine sévillane est célèbre pour avoir été l'une des rares autrices professionnelles reconnues au point d'avoir été à même de vivre des revenus de son écriture. Dans la pièce El Conde Partinuplés, l'argument mêle les enjeux de pouvoir de l'impératrice Rosaura et son désir d'accomplissement amoureux. Les interventions bénéfiques de la magicienne Aldora sont déterminantes et elles affectent chaque étape de l'évolution de l'action dramatique. Si l'on examine la nature du personnage d'Aldora, on peut y reconnaître aisément la plupart des éléments spécifiques des sorcières tels que les caractérisent les traités de démonologie. La dramaturge a, en outre, habilement tissé le lien intertextuel avec les modèles littéraires qui l'ont inspirée. Circé, Mélusine, Alcine ou Arminde sont régulièrement évoquées soit explicitement dans le discours dramatique soit suggérées par des analogies intertextuelles tant il est vrai, comme l'écrit Maria Aranda que « la figure de la magicienne [est] à la fois personnage tutélaire et principe poétique» $(2010: 62)$. De plus, dans cette comedia, la combinaison du rôle d'Aldora avec ceux de Rosaura, reine de Constantinople, et de Lisbella, héritière du trône de France, met en scène un magnifique trio féminin dont la pièce affiche le pouvoir invaincu. Cette affirmation d'une prééminence féminine n'a rien d'anodin venant d'une autrice, à une époque où la reconnaissance de ces plumes d'un autre genre, demeurait rare et incertaine. Les éléments discursifs et les actions scéniques en référence à la magie ou à la sorcellerie façonnent Aldora avec tous les ingrédients propres à la tradition, mais l'originalité du personnage tient à sa dimension allégorique, fortement liée au pouvoir de création par l'écriture. Le rôle de la magicienne irait-il donc au-delà de la tradition héritée de Circé ? Ana Caro semble s'appuyer sur ce personnage pour établir le passage fécond entre démon et démiurge. Le recours à la magie dans une perspective méta-théâtrale n'est-il pas alors l'indice d'une intention plus revendicatrice de la part d'une femme dramaturge en quête de reconnaissance et de légitimité ? À l'évidence, l'impact de ce personnage et du rôle qui lui est dévolu participe de la mise en œuvre d'une poétique du féminin dans la comedia d'Ana Caro.

\section{Argument de la pièce}

El Conde Partinuplés est l'une des deux seules comedias d'Ana Caro dont le texte est parvenu jusqu'à nous ${ }^{1}$. Elle fut publiée en 1653 dans le Laurel de comedias de diferentes autores de Diego de Balbuena. Inspirée de motifs de la légende arthurienne et du mythe 
de Cupidon et Psyché, l'intrigue est fondée sur une réélaboration du schéma traditionnel de l'examen de maris. Les différents prétendants à la main, et au trône, de Rosaura, doivent surmonter plusieurs épreuves pour que l'impératrice, que ses vassaux forcent à se marier pour assurer la pérennité de sa dynastie sur le trône, choisisse son futur époux. Rosaura, indécise et inquiète par une prophétie lui prédisant une trahison, fait appel à sa cousine et confidente, la magicienne Aldora. Les connaissances de cette dernière lui donnent le pouvoir de montrer à distance les différents prétendants à la main de la jeune reine. Rosaura tombe immédiatement amoureuse du comte Partinuplés, héritier du trône de France, et se met en quête d'obtenir son cœur alors qu'il est déjà engagé auprès de sa cousine, Lisbella. La dame obstinée sollicite encore l'entremise d'Aldora pour faire parvenir un portrait d'elle aux mains du comte. Follement épris de cette image, celui-ci se met en quête de la belle inconnue. Aldora, par ses actions magiques, guide ses pas depuis la France jusqu'au château enchanté de Rosaura situé près de Constantinople, où il pénètre malgré les craintes de son valet Gaulín. Enfin réunis sous le même toit, les amants s'y retrouvent dans l'obscurité, car l'impératrice a fait jurer au comte de ne pas chercher à l'identifier ni à la reconnaître. Le galant, soumis à ses désirs, lui obéit sagement. Mais, un soir, alors que Partinuplés fait le récit des batailles remportées pour sauver le trône de son oncle le roi de France, Rosaura s'endort subitement. Profitant de cette occasion, le comte, mu par une intense curiosité, éclaire les traits du visage de la belle endormie. Rosaura s'aperçoit de ce manquement à la parole donnée et s'estimant trahie, elle prononce, avec une violence inouïe, un verdict sans appel contre son amant malheureux qu'elle ordonne d'exécuter. Aldora, encore elle, prend l'initiative de le soustraire au courroux de la dame offensée et lui épargne la sentence fatale. Ainsi sauvé, il revient, le visage masqué, pour participer au tournoi des prétendants que l'impératrice a été contrainte d'organiser : le vainqueur de ces joutes sera son époux. Au même moment, Lisbella, arrive à la tête d'une armée pour libérer son cousin et futur roi de France qu'elle croit détenu par Rosaura. Les deux dames, rejointes par Aldora, assistent ensemble au tournoi et admirent les combats du comte qui, victorieux de tous ses rivaux, dévoile finalement son identité. Il confirme son amour pour Rosaura et sa décision de l'épouser. Il renonce définitivement au trône de France en faveur de sa cousine Lisbella qui donne sa main à Federico, l'héritier du royaume de Transylvanie, et Aldora est promise à Eduardo, prince d'Écosse.

4 Ce bref rappel de l'action dramatique permet de mieux appréhender l'importance et l'impact du rôle dévolu à la magicienne dans la pièce d'Ana Caro. De fait, c'est elle qui rend possible l'union des amants par l'étendue de ses pouvoirs et elle encore qui assure l'issue heureuse du conflit par la liberté de son initiative. La littérature espagnole en général et le théâtre du Siècle d'or en particulier offrent une intéressante galerie de personnages féminins dotés de pouvoirs magiques dont l'action facilite les rencontres amoureuses. Dans le cadre limité de cette étude, il serait fastidieux de citer les nombreux référents d'une dramaturgie de la sorcellerie qui ont précédé l'œuvre d'Ana Caro. Cervantès, Lope de Vega et ses épigones, Ruiz de Alarcón ou Mira de Amescua, et bien sûr Calderón de la Barca, ont tous été inspirés par cette matière qui permet de mettre en pratique la « recherche de l'effet" par l'efficacité du recours au surnaturel. En effet, l'admiratio procède de l'agencement cumulé d'un récit dramatique plein de surprises et d'une mise en scène ingénieuse et, comme l'écrit Jean Canavaggio, « d'une action capable de projeter le spectateur au-delà de lui-même et de l'arracher au quotidien » $(1977: 347)$. Nous ne rappellerons qu'un seul exemple lié à la figure qui 
nous intéresse ici : celui de Lope de Vega qui, non seulement a consacré un poème narratif au personnage de la magicienne en publiant LaCirce en 1624, mais en a également décliné les contours à travers des rôles complexes tels que celui de Fabia dans la pièce El caballero de Olmedo. Dans cette célèbre comedia, Tello, le serviteur du galant amoureux, fait un éloge remarquable des exceptionnelles qualités de la magicienne pour inciter son maitre à utiliser ses services. Il insiste notamment sur le fait que son savoir dépasse ceux de Circé, Médée et Hécate : « No supo Circe, Medea / ni Hécate lo que ella sabe ${ }^{2}$.» Cette citation a le mérite de réunir les trois figures majeures d'une généalogie de sorcières dont les portraits successifs ont permis l'élaboration d'un archétype fécond et fréquemment reproduit par la suite. Aldora, la magicienne conçue par Ana Caro dans sa pièce, s'inscrit de toute évidence dans cette lignée. Toutefois, l'autrice lui a conféré des pouvoirs amplifiés qui font évoluer ce personnage et lui confèrent une dimension emblématique du pouvoir féminin de création.

\section{Aldora, magicienne complice et bénéfique}

Dans les créations littéraires, on l'a dit, la figure de la sorcière présente un certain nombre de traits invariants, parfaitement codifiés, qui mêlent les accusations populaires les plus fréquentes concernant ces femmes et les récits diffusées par les hommes chargés de les juger (Caro Baroja, 1975 : 179-228). La typologie des aptitudes du personnage d'Aldora dans la pièce d'Ana Caro permet de retracer ce portrait ancien bien connu et d'y déceler les prémices d'une mythologisation de la figure de la sorcière telle qu'elle émerge des discours des juges ou des théologiens à cette époque charnière. Ainsi, dès les premières apparitions, Aldora semble-t-elle dotée de ce pouvoir exceptionnel d'aller et venir entre le monde des humains et celui des esprits. Elle correspond à cet agent intermédiaire qualifié par les auteurs du Malleus Maleficarum de «medium qui participe des extrêmes» (Institoris \& Sprenger, 1973 :141). Ce pouvoir d'intercession renvoie à une réalité redoutée et mystérieuse qu'elle partage avec des figures issues de la filiation d'Homère, dans le chant X de l'Odyssée et d'Ovide dans le livre XIV des Métamorphoses à laquelle s'ajoute ici une dangereuse proximité avec le diable. Dans la généalogie de Circé, particulièrement féconde, deux avatars de la littérature italienne de la Renaissance ont particulièrement marqué de leur influence les créations espagnoles du $\mathrm{XvII}^{\mathrm{e}}$ siècle: Alcine, dans le Roland furieux de l'Arioste en 1532 et Armide dans La Jérusalem délivrée du Tasse en 1581. Avec le personnage d'Aldora, Ana Caro semble à son tour s'inscrire dans cette filiation. Ainsi, la définition que Jean Rousset propose de Circé semble entrer en résonnance avec le personnage imaginé par Ana Caro :

Circé, c'est la magicienne qui d'un homme fait un animal et de nouveau un homme ; qui prête et retire à chacun tous les corps, toutes les figures, plus de visages, mais des masques; elle touche les choses, et les choses ne sont plus ce qu'elles étaient; elle regarde le paysage et il se transforme. Il s'emble qu'en sa présence l'univers perde son unité, le sol sa stabilité, les êtres leur identité ; tout se décompose pour se recomposer, entraîné dans le flux d'une incessante mutation, dans un jeu d'apparences toujours en fuite devant d'autres apparences. (1995:16)

Les différents traits propres à cette figure, devenue emblématique de l'esthétique et de la philosophie du baroque, sont parfaitement identifiables dans le personnage d'Aldora. 
va ainsi pouvoir regarder simultanément ses quatre prétendants grâce au truchement d'un miroir magique. La formule rituelle de l'incantation prononcée par Aldora est tout particulièrement intéressante. Elle rappelle l'origine hybride de son pouvoir où se mêlent le savoir antique de la magie héritée de Circé et l'image démoniaque de la sorcière issue de la tradition médiévale et de ses prolongements contemporains dans l'Espagne inquisitoriale (Jacques-Chaquin, 1978: 137-171). Le nom choisi par la dramaturge, Aldora, est en lui-même porteur d'un faisceau de suggestions. Il annonce l'essence du personnage si l'on considère que l'étymologie populaire peut facilement y entendre la contraction du syntagme ala dorada, «aile dorée ». Tout se passe comme si la dramaturge avait voulu ainsi rappeler, non sans humour, l'aptitude essentielle de cette magicienne, et de tant d'autres, à se déplacer dans les airs, et à transporter les personnages à une vitesse extraordinaire d'un lieu à un autre. De fait, Aldora préside à tous les déplacements du comte et de son valet, de même qu'elle est capable d'informer la reine de tout ce qui se passe loin du palais de Constantinople, dans des contrées lointaines. C'est elle, encore, qui organise l'ascension subite du comte et de son valet vers le ciel lui permettant ainsi d'échapper à la vindicte de la reine et au sort cruel qui lui était promis. Son action bénéfique sauve le galant de la mort annoncée. Au-delà de cette qualité de "déesse des voyages", le nom d'Aldora présente également une probable filiation intertextuelle avec Alcine, la belle magicienne amoureuse de Roger dans le Roland furieux d'Arioste. Le préfixe commun de leur nom [Al-] fonctionne pour le lecteur-spectateur comme un marqueur phonique: il évoque sa parenté avec cette lignée de femmes riches d'un savoir ancien qui maîtrisent les conjurations et les sortilèges. Toutefois, il faut noter que, dans la pièce d'Ana Caro, Aldora met toujours ses connaissances et son pouvoir au service de l'amour grâce à une action bénéfique. Le rôle de la magicienne est donc un adjuvant positif dans le schéma actantiel de la pièce.

8 Si Aldora a la capacité d'abolir les distances, on notera qu'elle exerce tout d'abord ce pouvoir grâce à un miroir magique qui sert littéralement de "télévision " puisqu'il permet de voir à distance. Elle énonce sa proposition pour aider Rosaura dans le choix de son futur époux après que cette dernière a fait part, dans une tirade désespérée, du conflit dans lequel elle se débat :

\begin{tabular}{|l|l|l|}
\hline ALDORA: & $\begin{array}{l}\text { Lo más que hoy puedo hacer } \\
\text { por ti, pues sabes mi ingenio } \\
\text { en cuanto a la mágica arte, } \\
\text { es enseñarte primero } \\
\text { en aparentes personas } \\
\text { estos príncipes propuestos }\end{array}$ \\
\hline
\end{tabular}

Effectivement, peu après, la magicienne met à exécution ce projet et elle va permettre à Rosaura de voir les images des différents prétendants princiers, de façon simultanée et à distance, chacun dans leurs activités comme une capture d'écran instantanée de leur réalité quotidienne. Dans cette action primordiale, elle est la médiatrice de l'amour et elle suscite l'éveil de la passion au point d'en rendre dépendant le personnage qui l'éprouve. Les conjurations et les sortilèges vont appuyer cette stratégie de médiation entre les ordres naturels masculins et féminins. Aldora est l'élément actif d'une magie à finalité érotique qui l'installe, au départ de l'action, dans le rôle traditionnel de l'entremetteuse. Elle appartient à la catégorie définie par Julio Caro Baroja, dans son 
ouvrage majeur, Las brujas y su mundo, comme "hechicera mediadora " ou magicienne médiatrice (Caro Baroja, 1986 : 55). Ana Caro, par la subtilité suggestive de son écriture dramatique, met en évidence l'impact spectaculaire de la médiation exercée par le personnage d'Aldora. Elle s'assure de son efficacité visuelle et sonore en s'appuyant autant sur la gestuelle associée à l'observation des images que sur le pouvoir de conviction de la parole. L'objet transitionnel du miroir focalise les regards de Rosaura et des spectateurs dans ce spectacle dans le spectacle, et tout le monde est entrâné dans cette réalité parallèle virtuelle. Un «allí » ou "là-bas » est ainsi suggéré hors scène, une sorte d'ailleurs lointain qui prolonge l'espace scénique et agrandit l'espace dramatique :

\begin{tabular}{|c|c|}
\hline ALDORA: & $\begin{array}{l}\text { El que allíse ve suspenso } \\
\text { o entretenido mirando } \\
\text { el sol de un retrato bello, } \\
\text { es Partinuplés famoso, } \\
\text { de Francia noble heredero } \\
\text { por sobrino de su Rey, } \\
\text { que le ofrece en casamiento } \\
\text { a Lisbella prima suya; } \\
\text { príncipe, noble, modesto, } \\
\text { apacible y cortesano, } \\
\text { valiente, animoso y cuerdo. } \\
\text { Éste es más digno de ser } \\
\text { de entre los demás, tu dueño, } \\
\text { a no estar, como te he dicho, } \\
\text { tratado su casamiento } \\
\text { con Lisbella. }\end{array}$ \\
\hline ROSAURA: & $\begin{array}{l}\qquad \text { ¿Con Lisbella? } \\
\text { Por eso, Aldora, por eso } \\
\text { me lleva la inclinación } \\
\text { aquel hombre. }\end{array}$ \\
\hline ALDORA: & $\begin{array}{l}\text { Impedimento } \\
\text { tiene, a ser lo que te digo. }\end{array}$ \\
\hline ROSAURA: & $\begin{array}{l}\text { ¡Ay Aldora! A no tenerlo, } \\
\text { otro me agradara, otro } \\
\text { fuera, en mi grandeza empeño } \\
\text { de importancia su elección; } \\
\text { pero, si le miro ajeno, } \\
\text { ¿cómo es posible dejar, } \\
\text { por envidia o por deseo, } \\
\text { de intentar un imposible, } \\
\text { aun siendo sus gracias menos }{ }^{4} \text { ? }\end{array}$ \\
\hline
\end{tabular}

Le désir de Rosaura pour le comte est donc induit par la médiation de Aldora qui lui vante les qualités du sujet ainsi offert à son regard. La convoitise du bien d'autrui éveille souvent le désir amoureux et c'est le cas ici puisque, dès sa présentation, on 
annonce l'engagement du galant auprès de sa cousine Lisbella. Le recours à une scénographie rituelle dans la démarche d'Aldora intensifie le désir de la dame pour le comte qui est offert à son regard. Afin de procéder à cette opération, elle prononce une formule magique et s'adresse ainsi directement aux puissances infernales: «Espíritus infelices / que en el espantoso Reino / habitáis, por esas negras / llamas sin luz y con fuego / os conjuro, apremio y mando [... $]^{5}$.» Il est intéressant de noter qu'une formule identique est utilisée dans le théâtre bref de Cervantès, par Chanfalla, le rusé montreur de marionnettes de l'intermède El retablo de las maravillas, et cette similitude va au-delà de la simple répétition d'un discours rituel codifié (González, 2016 : 195-212). Les termes de l'incantation mettent en évidence le pouvoir verbal de la conjuration et, conformément aux traditions des rites magiques, le support visuel associé à la requête faisant émerger de la sorte un récit second et de nouveaux personnages. Ainsi dramatisée et ritualisée, la visualisation de ces images renforce leur impact sur le personnage de Rosaura. Aldora, intermédiaire d'une redoutable efficacité, est aussi l'agent de l'accélération de l'embrasement amoureux : elle fait un portrait très flatteur du galant, observé pour la première fois. Si à peine quatre vers suffisent à caractériser ses futurs rivaux, les princes de Pologne, d'Écosse et de Transylvanie, elle s'applique à préciser dans un développement encomiastique de seize vers les circonstances et les qualités qui font de Partinuplés le meilleur des prétendants et le plus sûr des époux. Le pouvoir du verbe est l'un des traits définitoires de la sorcière et l'impact performatif de ses répliques dans le dialogue dramatique produit un effet immédiat.

11 Plus tard, pour concrétiser les effets réciproques de cet amour au premier regard, Aldora fait parvenir entre les mains du comte un portrait peint de Rosaura. Le galant s'éprend immédiatement de ce beau visage et il n'a de cesse que de le retrouver. Il se lance à la poursuite d'une bête fauve, comme ensorcelé par ce monstre, comme l'indique la didascalie qui détaille l'apparition soudaine de la belle Rosaura ainsi métamorphosée :

Sale el CONDE tras una fiera vestida de pieles, vale dar y vuélvese una tramoya, y aparece ROSAURA, como está pintada en el retrato 6 .

12 Le comte se laisse alors envoûter par une formule obsédante par laquelle la belle métamorphosée en bête l'incite à la suivre : «Si me buscas, me hallarás. » La dame, aidée de la magicienne, l'attire dans l'épaisse forêt avec ce leitmotiv incantatoire (Montousse Vega, 1994 : 7-27). La scène n'est pas sans rappeler certains épisodes du Roland furieux de l'Arioste et, plus particulièrement, le trio formé par Roger, Bradamante et Alcine. Le personnage de Gaulín, valet du comte, est d'ailleurs chargé d'expliciter cette référence qui mobilise la relation intertextuelle entre les deux magiciennes Alcina et Aldora. Au cours de cette poursuite, où se succèdent les prodiges qui exaspèrent et inquiètent le valet, il s'exclame : "Orlando furioso, tate, / cada loco con su tema [...] $]^{7}$. Il convient aussi de rappeler que, si la poursuite dans la forêt est un motif fréquemment mobilisé dans les comedias, comme dans l'acte I de la pièce d'Ana Caro, il est traité ici dans une perspective peu habituelle. Si Aldora, comme Circé, sait transformer les humains en animaux, ici la dame métamorphosée en bête sauvage semble diriger les pas de l'amantchasseur. Ainsi, la menace change de camp. Le comte n'a plus qu'à avouer son impuissance en alternant les oxymores dans une réplique qui met en évidence sa passion : 


\begin{tabular}{|l|l|l|}
\hline CONDE: & $\begin{array}{l}{[. .]} \\
\text { Y si fiera cruel das vida, } \\
\text { beldad piadosa, das muerte. } \\
\text { ¿Eres de este valle diosa, } \\
\text { eres ninfa de este monte }\end{array}$ \\
& \\
&
\end{tabular}

On le voit l'asservissement du galant à son désir est total et le pouvoir de la magicienne semble tacitement accepté par le couple des amants qui en tire tous les bénéfices. Cependant, a contrario, il éveille toutes les peurs ancestrales chez le valet Gaulín. La couardise et la poltronnerie traditionnelles de cet emploi exacerbent la vulnérabilité du gracioso face à ces croyances. À la fin de l'acte I, alors que Gaulín et son maître sont perdus dans la forêt, sous l'orage, et menacés par d'étranges bêtes sauvages dotées de la parole, le valet exprime sa crainte de se trouver face aux héritières des sorcières de Thessalie ou à Circé elle-même: "Si es Tesalia, ola engañosa / de Circe... estancia agradable 9 . La dimension intertextuelle de l'allusion évoque une réaction populaire presque atavique. Si les répliques de Gaulín font souvent sourire, comme dans l'énumération insolite par laquelle il tente de définir la dame invisible, «Bruja, monstruo o cocodrilo / será pues tanto se esconde $e^{10} "$, elles renvoient aussi à une terreur ancienne aux prolongements tristement contemporains dans l'Espagne du XvII ${ }^{e}$ siècle. Une telle hybridité est donc l'essence même du personnage féminin ainsi protégé par la magie, qui la rend puissante: mi-ange et mi-bête, mi-déesse et mi-démon. Son pouvoir d'attraction irrésistible est renforcé par l'énigme d'une apparence monstrueuse, et il s'inscrit pleinement dans la tradition mythique des femmes de pouvoir, dans la lignée de Circé et de Mélusine (Montandon, 2018). C'est bien Aldora qui a façonné cette double image de la femme et du monstre et qui rend Rosaura capable de fasciner le galant ainsi dominé.

Comme cela a déjà été évoqué, la magicienne organise les transports des personnages d'un pays à l'autre. Ces nombreux déplacements se font de la manière la plus extraordinaire comme le souligne, au début de l'acte II, une réplique de Gaulín qui exprime sa stupéfaction devant tant de prodiges :

\begin{tabular}{|l|l|}
\hline GAULÍN: & $\begin{array}{l}\text { Con eso me desbautizo, } \\
\text { me enfurezco, me remato } \\
\text { ¿no ves que fue rudo hechizo? } \\
\text { Pues luego ver una fiera } \\
\text { y transformarse en mujer, } \\
\text { (aunque no hay mucho que hacer) } \\
\text { iquién sino el diablo lo hiciera? } \\
\text { Entrarnos en un navío } \\
\text { desarbolado y al punto } \\
\text { verlo con jarcias, pregunto } \\
\text { ¿quién pudo hacerlo, amo mío? } \\
\text { No ver quién lo gobernaba, } \\
\text { quién, cosa y cómo guió } \\
\text { hasta aquí, pregunto yo, } \\
\text { iquién lo hizo, Señor }{ }^{11} ?\end{array}$ \\
\end{tabular}



mauvais esprit ou du diable lui-même, comme il le dit un peu plus loin, abasourdi par la disparition insolite des objets sur la table du dîner qui les attendait : «Y esto, ¿no lo hace el diablo ${ }^{12}$ ?»

Le repas au château correspond d'ailleurs à un autre motif qui relie Aldora à la généalogie circéenne. Il a lieu dans un palais enchanté, véritable royaume de l'illusion et du paraître dans des scènes d'une grande efficacité dramaturgique. Devant ses hôtes abasourdis, la magicienne fait apparaître et disparaitre des objets avec malice pour les maintenir sous son emprise devant tant de faits inexpliqués. Ana Caro s'appuie sur cette dramaturgie de l'invisibilité dont les effets sont assurés pour la captation des spectateurs amusés. La disparition des plats au nez et à la barbe du bouffon, gourmand et couard, a un effet comique immédiat :

\begin{tabular}{|l|l|l|}
\hline & & Apártele una empanada a una esquina de una mesa. \\
\hline GAULÍN: & $\begin{array}{l}\text { Dios te de vida; que a fe, } \\
\text { que la deseaba ya. }\end{array}$ \\
\hline & & Al tomarla, ábrela y salen cuatro o seis pájaros vivos de ella. \\
& $\begin{array}{l}\text { ¿Qué es esto? Burla excusada; } \\
\text { luego que empanada vi, } \\
\text { por Dios vivo que temí } \\
\text { que me daban en pan nada }\end{array}$ \\
\hline
\end{tabular}

17 Comme ces déclinaisons de traits caractéristiques le confirment, la plupart des spécificités historiques et des attributs mythiques de la sorcière se retrouvent dans le personnage d'Aldora.

Mais, comment ne pas envisager un impact plus profond derrière ces plaisanteries et ces jeux de mots? Gaulín, le valet gracioso est traditionnellement le complice du spectateur. Celui-ci ne serait-il pas lui-même terrifié ou, à tout le moins, troublé par l'évocation de ces traditions ancestrales? Plusieurs détails jouent sur le double mouvement d'attraction et de répulsion qu'engendraient ces phénomènes. Ainsi, les nombreux récits de sabbats largement diffusés à cette époque faisaient régulièrement mention de la faim persistante des participants au sortir de la table orgiaque. Cet invariant des récits est considéré comme la preuve, pour Henri Boguet au début du XVII siècle, dans le Discours exécrable des sorciers, de l'intervention du démon : «[...] d'où il se voit que le Diable est toujours trompeur, puisqu'il repaist les siens de vent. » $(1980: 27)$ Dans la pièce d'Ana Caro, la finalité de tous ces sortilèges est officiellement le choix éclairé de l'époux futur de la reine Rosaura mais, en fait, c'est l'accomplissement du désir amoureux qui semble avant tout être le moteur de cette quête. Or, les ébats nocturnes entre la dame et son galant laissent envisager une sensualité parfaitement épanouie et cette harmonie est suggérée à plusieurs reprises. Outre l'obscurité qui est de mise, ces moments sont marqués par le mystère qui entoure l'identité de la belle dont le visage demeure caché au regard de l'amant. Le dialogue, par le détour du comique, évoque une forme de diabolisation érotique du corps de la femme devenue 
" ange des ténèbres" dans une réplique suggestive du valet Gaulín toujours porteur d'une parole misogyne conforme aux attentes majoritaires des spectateurs :

\begin{tabular}{|l|l|}
\hline & \multicolumn{1}{c|}{$\begin{array}{c}\text { Señor, ioyes?, } \\
\text { algo tienen de Noruega } \\
\text { estos oscuros amores, } \\
\text { pues de la luz de tus días } \\
\text { nos gozas más de las noches. } \\
{[. . .]}\end{array}$} \\
& $\begin{array}{l}\text { ¿cómo el ángel de las tinieblas } \\
\text { no sale a hacerte favores }{ }^{14} ?\end{array}$ \\
\hline
\end{tabular}

\section{Aldora, démon et démiurge}

19 À la fin de la pièce, la magicienne Aldora est le véritable agent de la résolution du conflit. À l'évidence, elle forme avec la reine, depuis le début de l'action, un couple complémentaire. Aldora autorise le dénouement heureux grâce à son initiative indépendante qui permet le retour en scène et en lice du comte pour le tournoi final alors qu'il avait été condamné à mort par Rosaura. Aldora a su anticiper et enfreindre l'ordre de la dame offensée en choisissant de laisser la vie sauve au galant en dépit de la sentence fatale. Elle a ensuite caché et protégé le comte, à l'insu de la reine, pour l'amener, au moment opportun, à combattre dans le tournoi décisif. Quand Partinuplés réapparaît pour se mesurer à ses rivaux, la reine ignore ce qui se trame et ne peut le reconnaître puisque son visage est occulté par le heaume. Cette fois, le masque a changé de support et le renversement des rôles est, de fait, propice au rétablissement des fonctions habituellement attribuées à chaque genre dans la tradition. Le personnage masculin, pourvu de cet accessoire qui le cache, redevient actif autant qu'acteur, devant la tribune des dames, attentives spectatrices qui assistent à la scène finale.

C'est Aldora qui a imaginé et mis en scène tous les rebondissements d'une action dont la mobilité est toujours mise au service du double dessein de sa reine, conserver son royaume et $\mathrm{y}$ vivre auprès d'un époux aimé. Dès lors, l'analogie entre Aldora et Chanfalla, le montreur de marionnettes du Retablo de las maravillas de Cervantès s'impose dans toute sa pertinence. Elle est d'abord fondée sur la réappropriation d'un discours rituel dont José María Díez Borque a mis en évidence le fonctionnement poétique (1985: 47-87). L'incantation s'appuie, en effet, sur des combinaisons structurelles récurrentes, telles que le rythme ternaire qui en amplifie la portée déclamatoire : «os conjuro, apremio y mando... » Mais elle permet aussi la mise en place d'une poétique de fusion des plans qui superpose le surnaturel au « réel » du récit fictif. En outre, Ana Caro prolonge ce propos par une perspective méta-théâtrale efficacement portée par le contexte. En effet, si le personnage d'Aldora donne à voir les personnages de la pièce avant de les mettre en scène en écrivant leur histoire, si elle est aussi la garante de la fin heureuse puisqu'après avoir agi sur la dame, elle guide les pas et oriente l'action du galant pour assurer le dénouement du conflit, tout concourt à présenter la magicienne comme l'agent essentiel de la pièce qui se joue et dont elle 
contribue à construire l'action. Elle assume donc ici la fonction d'autrice en engendrant le récit dramatique et également celle de metteuse en scène en usant de stratégies et de stratagèmes qui en rendent le déroulement spectaculaire.

Dans la pièce d'Ana Caro, la magie est consubstantielle à la trame. Le bref dialogue qui suit, entre le valet Gaulín et la magicienne Aldora, au-delà de l'humour immédiat des répliques, laisse percevoir le positionnement idéologique de la dramaturge dans la pièce :

\begin{tabular}{|l|l|l|}
\hline & & Sale ALDORA, en una apariencia, en que se subirán con ella los dos al fin del paso. \\
\hline & ALDORA: & ¿Conde? ;ah, Conde! \\
\hline & CONDE: & ¿Quién me llama? \\
\hline & ALDORA: & Yo soy. \\
\hline & GAULÍN: & $\begin{array}{c}\text { Tramoya tenemos; } \\
\text { esto es hecho. }\end{array}$ \\
\hline & CONDE: & ¿Oiste hablar? \\
\hline & & En el aire, sin verse ${ }^{15}$. \\
\hline
\end{tabular}

La dramaturgie combine ici l'effet comique d'un dialogue vif aux répliques brèves avec un jeu scénique à la gestuelle efficace où se répondent la surprise du maître et la peur du valet. Mais, il ressort surtout de ce passage que le pouvoir de la magicienne est explicitement comparé à celui de la dramaturge. L'allusion méta-théâtrale fait référence aux deux dispositifs cités dans l'extrait ci-dessus, la "apariencia ${ }^{16}$ " et la " tramoy $a^{17}$ » en mettant ainsi sur le même plan la technique qui, grâce à la machinerie, permet l'élévation des personnages dans les airs, et la magie, qui autorise les déplacements extraordinaires organisés par une enchanteresse non seulement habile mais astucieuse. Comme le rappelle Julio Caro Baroja : «La palabra tramoya tiene varias acepciones, como es sabido: la tramoya es una máquina para figurar transformaciones en el teatro, o el conjunto de ellas. Pero también se llama así a un enredo bien urdido. » (1974:34) Cette magie est aussi bien celle du théâtre et de ses techniques novatrices, dont Ana Caro paraît ici revendiquer l'efficacité, que celle que porte le personnage d'Aldora chargée de les mettre en pratique pour les fins souhaitées. La revendication de la dramaturge est double : l'écriture défend explicitement le «teatro de tramoyas » et, en même temps, elle suggère implicitement l'ampleur du pouvoir invaincu des femmes qui, dans la pièce, grâce au savoir et à l'action d'Aldora, n'a d'égal que celui de l'autrice dont elle est la figure spéculaire.

Dans le dénouement heureux de la scène finale, les personnages féminins semblent se soumettre à l'ordre patriarcal incarné par le comte. La convention théâtrale des mariages multiples est ainsi respectée. Toutefois, les répliques des futures épouses interrogent par leur extrême brièveté. Elles deviennent monosyllabiques et l'acceptation tacite de ce nouvel état se fait sans exaltation ni enthousiasme apparent. Sans doute serait-il possible de mettre en relation ce comportement avec les aveux a minima consentis par les sorcières lors des interrogatoires, ultimes recours de leur 
rébellion intime, tels que les rapporte Michelet dans les exemples de son ouvrage La sorcière et notamment celui de la Cadière (1862: 310-377). Plus récemment, Nicole Jacques-Chaquin (1978) a tenté de déchiffrer la portée de cette attitude qu'elle désigne par le concept de "taciturnité » en analysant le discours perdu des sorcières tel qu'il est rapporté par le pouvoir dominant, les médecins, les théologiens et les historiens. Les femmes puissantes de la pièce d'Ana Caro semblent ainsi consentir à se mettre en conformité avec le système dominant et ce silence relatif qui caractérise leurs dernières répliques occulte prudemment leur pouvoir véritable.

La figure d'Aldora dans la pièce d'Ana Caro s'inscrit avec profit dans la généalogie de Circé. La magicienne est le truchement nécessaire à l'aboutissement heureux du conflit amoureux. Par son habileté à mobiliser les artifices magiques, elle autorise des projections visuelles et physiques qui correspondent pleinement à l'esthétique baroque. On y retrouve les jeux d'opposition de plans fondés sur la dialectique illusion/ désillusion, ou essence/apparence, si féconde au théâtre et particulièrement mise à profit par les auteurs du XVII espagnols contemporains d'Ana Caro. Jean Rousset résume ainsi cette problématique sous-jacente :

[...] toute cette fluidité dans les personnages et les situations implique une psychologie de l'intermittence et de la mobilité ; l'être ne se saisit que dans le reflet fugitif de ses apparences et ne se donne qu'en se dérobant. (1995: 44)

Ana Caro offre une dimension supplémentaire au rôle de la magicienne qui, dans cette pièce, organise la mobilité et en contrôle tous les effets sur l'action dramatique. C'est elle encore qui, in fine, fait tomber les masques pour que l'amour ne soit plus un piège illusoire mais une étape vers la vérité de chacun. C'est là un écart significatif par rapport à la tradition littéraire dans laquelle s'inscrit le personnage qui confère une dimension intéressante à cette comedia de femmes, écrite par une femme. Cette différence est majeure par rapport aux modèles intertextuels que furent les magiciennes de l'Arioste et du Tasse, Alcine et Armide. Elle consiste à offrir au lecteurspectateur un dénouement heureux ourdi par la magicienne qui en fait partie intégrante. Alors que Alcine disparait du récit lorsque son vrai visage apparaît et que, de la même façon, Armide doit se convertir et renoncer à l'amour, Aldora mène à terme son projet, réunit les amants et fait partie des heureuses élues qui forment les unions de la scène finale.

Ana Caro a choisi de mettre en évidence la victoire finale d'un trio féminin parvenu à ses fins : Aldora épouse Eduardo, en même temps que Rosaura se donne au comte et Lisbella promet sa main à Roberto. Les mariages multiples qui clôturent la pièce ne sont pas ici seulement un motif structurel des comedias nuevas, imitées du modèle de Lope de Vega. Ils constituent l'aboutissement de l'action bénéfique d'Aldora au profit de Rosaura. En sauvant la vie du comte, elle anticipe son retour dans l'action et organise les circonstances de sa victoire finale sur ses rivaux, tout en garantissant à l'impératrice son maintien sur le trône.

De la sorte, la revanche de Circé est ainsi actée par la fin euphorique de la pièce. On est fondé à penser qu'elle sous-tend une autre forme de revanche... On est fondé à penser que l'intention implicite de la dramaturge et autrice dépasse le divertissement traditionnel pour suggérer une interprétation plus idéologique. Par le détour de la fiction dramatique, la victoire des personnages féminins de la pièce, grâce à l'action d'une magicienne ingénieuse, invite habilement à considérer la nécessaire reconnaissance du pouvoir féminin dans le champ de la création littéraire. 


\section{BIBLIOGRAPHIE}

ARANDA Maria (2010), « De Circé à Ulysse. Le naufragé "gothique” de Lope de Vega », Bulletin Hispanique, 112(1), 61-73.

Boguet Henry (1980), Discours exécrable des sorciers [1602], éd. de Nicole Jacques-Chaquin et Philippe Huvet, Paris: Le Sycomore.

CANAVAGGIO Jean (1977), Cervantès dramaturge, un théâtre à naître, Paris : Presses universitaires de France.

CARo BAROJA Julio (1974), Teatro popular y magia, Madrid : Revista de Occidente.

Caro Baroja Julio (1975), " Arquetipos y modelos en relación con la historia de la brujería », Brujología. Congreso de San Sebastián, Madrid : Seminarios y Ediciones, 179-228.

CARo BARoja Julio (1986), Las brujas y su mundo, Madrid : Alianza.

CARo de Mallén Ana (1993), El Conde Partinuplés, éd. de Lola Luna, Kassel : Reichenberger.

DíEZ BoRQuE José María (1985), « Conjuros, oraciones, ensalmos...: formas marginales de poesía oral en los Siglos de Oro », Bulletin Hispanique, 87(1-2), 47-87.

Gil-Albarellos Susana \& Rodríguez PequeÑo Mercedes (dir.) (2006), Ecos silenciados: la mujer en la literatura española. Siglos XII al XVIII, Junta de Castilla y León.

GoNZÁLEZ Aurelio (2016), « Conjuro, magia y demonios en el teatro cervantino », M. L. Lobato, J. San José \& G. Vega (dir.), Brujería, magia y otros prodigios en la literatura española del Siglo de Oro, Alicante : Biblioteca Virtual Miguel de Cervantes, 195-212.

INSTITORIS Henri \& SPRENGER Jacques (1973), Le marteau des sorcières [Malleus Maleficarum, 1486], édition et traduction d'Armand Danet, Paris : Plon.

JACQUES-CHAQUIN Nicole (1978), « Le maléfice de taciturnité : esquisse d'une étude du mythe de la sorcière ", Cahiers de Fontenay, 9-10, 137-171.

JACQUES-CHAQUIN Nicole (2002), « Figures de sorcières : mythe et individualités », C. Planté (dir.), Sorcières et sorcelleries, Lyon : Presses universitaires de Lyon, 65-80, <http://

books.openedition.org/pul/7178>.

LOPE DE VEGA (2000), El caballero de Olmedo [1620], éd. de Francisco Rico, Madrid : Cátedra, Letras Hispánicas, 147.

MicheLET Jules (1964), La sorcière [1862], Paris : Julliard. [Version de 1878 numérisée sur <https:// gallica.bnf.fr/ark:/12148/bpt6k57265106.texteImage\#>.]

MontAndon Alain (2018), Mélusine et Barbe-Bleue. Essai de sociopoétique, Paris : Honoré Champion.

MontousSE VeGA Juan Luis (1994), « “Si me buscas, me hallarás”: la configuración del discurso femenino en la comedia de Ana Caro El Conde Partinuplés », Archivum, 40-45(2), 7-27, <www.unioviedo.es/reunido/index.php/RFF/article/viewFile/492/481>.

RouSSET Jean (1995), La littérature à l'âge baroque en France. Circé et le paon, Paris : J. Corti.

ROUANE SOUPAULT Isabelle (2021), “ Une si vertueuse audace... » Les femmes dramaturges dans l'Espagne $d u$ XVII ${ }^{e}$ siècle, Aix-en-Provence : Presses universitaires de Provence. [Traduction de la pièce Le comte Partinuplés, p. 210-256.] 


\section{NOTES}

1. Ana Caro de Mallén, El Conde Partinuplés, éd. de Lola Luna, Kassel : Reichenberger, 1993. Édition de référence. Pour la traduction en français, voir Isabelle Rouane Soupault, "Une si vertueuse audace..." Les femmes dramaturges dans l'Espagne du XVII siècle, Aix-en-Provence: Presses universitaires de Provence, 2021, p. 211-256.

2. Lope de Vega (2000: III, v. 1922-1923, 181). Traduction: «Ni Circé, ni Médée et pas même Hécate n'ont su tout ce qu'elle sait. »

3. Ana Caro de Mallén (1993 : 91, v. 293-298). Traduction: «Tout ce que je peux faire pour toi aujourd'hui, car tu sais combien je suis experte dans l'art de la magie, c'est d'abord de te montrer l'apparence physique de ces princes qui te sont proposés. »

4. Ana Caro de Mallén (1993 : 95, v. 356-384). Traduction : «Ce dernier serait, plus que tous les autres, le plus digne de devenir ton seigneur et maître, si comme je te l'ai dit, son union n'était pas déjà préparée et s'il n'était pas promis à Lisbelle. - ROSAURE : À Lisbelle ? C'est donc cela, Aldore, oui c'est cela, sans aucun doute, qui entraîne mon penchant pour cet homme-ci. - ALDORE : Je te le dis à nouveau, il y a là un empêchement majeur. - ROSAURE : S'il n'y en avait point eu, un autre m'aurait plu, un autre aurait été l'objet de mon choix obstiné. Mais, quand je le vois ainsi appartenir à une autre, et même si ses attraits sont moins nombreux, comment pourrai-je, ne pas envisager de tenter l'impossible, que je sois guidée par l'envie ou bien par le désir. »

5. Ana Caro de Mallén (1993 : 93-94, v. 323-326). Traduction : « Vous, âmes en peine qui vivez dans l'effrayant royaume des ombres, entourées des flammes obscures et brûlantes à la fois, je vous invoque, je vous conjure et je vous ordonne [...]. »

6. Ana Caro de Mallén (1993: 105). Traduction : «Le comte court derrière un fauve dont on ne voit que les peaux de bêtes, il est sur le point de le frapper lorsque la machinerie tourne et Rosaure apparait telle qu'elle est peinte sur le tableau. »

7. Ana Caro de Mallén (1993:110, v. 686-687). Traduction: «On croirait vraiment entendre Roland le Furieux ? À chaque fou, sa marotte !»

8. Ana Caro de Mallén (1993 : 107, v. 623-626). Traduction : « [...] et si, quand vous êtes une bête cruelle vous décidez de me laisser en vie, c'est la déesse bienveillante en vous qui me donnera la mort. Êtes-vous la déesse de ces vallées ? Êtes-vous la nymphe de ces montagnes?»

9. Ana Caro de Mallén (1993:111-112, v. 715-716). Traduction : "Si cette femme est Médée ou Circé la trompeuse... voilà qui nous promet un agréable séjour. »

10. Ana Caro de Mallén (1993:129, v. 1070-1071). Traduction: "Sûrement une sorcière, un monstre ou un crocodile sans doute, puisqu'elle tient tant à se cacher. »

11. Ana Caro de Mallén (1993 : 116, v. 787-801). Traduction : "Alors là, je suis accablé, furieux, totalement anéanti. Ne voyez-vous pas qu'il y a de la magie dans cette affaire? Qu'une bête sauvage, à peine entrevue, devienne d'un seul coup une femme (même s'il est vrai que l'une et l'autre se ressemblent beaucoup), qui peut le faire, si ce n'est le diable? Que nous entrions dans un navire sans mât et que, d'un seul coup, il retrouve tous ses gréements, je pose la question mon maître, qui donc peut le faire? Ne voir personne à la barre, personne tenir le gouvernail, ni savoir comment il est arrivé jusqu'ici, je repose la question, qui l'a fait, Monsieur?»

12. Ana Caro de Mallén (1993 : 120, v. 891). Traduction : «Et tout cela? N’est-ce point l'œuvre du diable?»

13. Ana Caro de Mallén (1993 : 122, v. 919-924). Traduction : "Le comte prend un plat posé dans un coin opposé de la table et le rapproche de Gaulin. Il contient un vol-au-vent. - GAULIN : Que Dieu t'accorde une longue vie, par ma foi, j'en mourais d'envie! En le prenant, il soulève le couvercle en croûte et trois ou quatre oiseaux en sortent vivants. Quoi ? Qu'est-ce? Encore une farce? Dès que j'ai aperçu les vol-au-vent, aussi vrai que je vous vois, je me doutais bien que je n'aurais que du vent et que tout le reste s'envolerait [...]. » 
14. Ana Caro de Mallén (1993:151, v.1555-1565). Traduction: «Entendez-vous, Monsieur? Ma foi, ces amours nocturnes ont quelque chose de polaire à force d'être toujours dans l'obscurité... On dirait que vous ne pouvez jouir que la nuit de la lumière de vos jours ! [...] Il fait nuit noire à présent. Comment se fait-il que le bel ange de ces ténèbres ne soit pas encore venu vous offrir ses faveurs?»

15. Ana Caro de Mallén (1993 : 163). C'est moi qui souligne. Traduction : «- ALDORE : Ah, comte, enfin, je vous retrouve... - COMTE : Qui m'appelle? - ALDORE : C'est moi. - GAULIN : Vous, Madame, est-ce l'effet de la machinerie ou d'une machination? - COMTE : As-tu entendu parler? - ALDORE : Comte, s'il vous plaît... Elle est suspendue en l'air et on ne la voit pas. »

16. On nommait « apariencia » le dispositif visuel, le plus souvent sur des toiles peintes ou sur des mannequins armés sur des poulies, permettant de faire apparaître et disparaître les personnages. Ce terme technique n'a pas de traduction précise en français.

17. Le terme "tramoya » est couramment traduit par machinerie, car il indique l'ensemble des dispositifs nécessaires aux changements de décors et à la mise en place des différents effets scéniques.

\section{RÉSUMÉS}

Le personnage de la magicienne Aldora dans la pièce d'Ana Caro El Conde Partinuplés, écrite en 1653, s'inscrit dans la lignée littéraire issue de Circé et des modèles italiens bien connus d'Alcine de l'Arioste ou d'Arminde du Tasse. La dramaturge sévillane s'appuie sur les motifs propres à cette tradition, déclinée par de nombreux auteurs du Siècle d'or espagnol, identifiables dans l'argument de sa pièce où ils sont enrichis des descriptions issues des procès inquisitoriaux. De l'invocation initiale des puissances infernales, aux sortilèges amoureux en passant par la métamorphose animale ou les déplacements extraordinaires, Aldora coïncide avec le type de la sorcière attendue par le public du théâtre. Toutefois, Ana Caro en amplifie la portée en accordant à son personnage le pouvoir de dénouer le conflit dramatique. Cette heureuse issue met en évidence la volonté de la dramaturge d'imposer la suprématie d'un trio de personnages féminins invaincus formé par Aldora et les deux dames puissantes que sont les reines Rosaura et Lisbella. De plus, l'homologie structurelle entre la figure de la magicienne et celle de l'autrice, soulignée par de fréquentes et drôles allusions méta-théâtrales dans le dialogue, est symboliquement porteuse d'une intention subversive qui éclaire les revendications de la dramaturge et sa quête de reconnaissance.

El personaje de la maga Aldora en la comedia de Ana Caro El conde Partinuplés, escrita en 1653, forma parte del linaje literario nacido con Circe y renovado con los exitosos modelos italianos de Alcina del Ariosto y Arminda del Tasso. La dramaturga sevillana se apoya sobre los motivos propios de esta tradición, ilustrada por numerosos autores del Siglo de Oro español, que va desarrollando en el argumento de su pieza y que se ven aumentados por los testimonios de los juicios inquisitoriales. Desde el conjuro inicial con el que convoca las potencias infernales hasta los sortilegios amorosos, la metamorfosis animal o los viajes extraordinarios, Aldora coincide perfectamente con el tipo de bruja o maga esperado por el público del teatro. Sin embargo, Ana Caro parece amplificar su dimensión al otorgar a su personaje el poder de desenredar y resolver el conflicto dramático. Este feliz desenlace pone de manifiesto la voluntad de la dramaturga de imponer la supremacía de un trio de personajes femeninos invictos formado por Aldora y las dos 
damas potentes que son las reinas Rosaura y Lisbella. Además la homología estructural entre la figura de la maga y de la autora, a las que hacen a menudo hincapié en el diálogo divertidas alusiones metateatrales, lleva simbólicamente una carga subversiva que aclara las intenciones de la dramaturga y su búsqueda de reconocimiento.

The character of the magician Aldora in Ana Caro El Conde Partinuplés's play, written in 1653, is part of the literary lineage of Circe and the well known Italian models of Alcina from Arioste or Tasso's Arminda. The Sevillian playwright relies on the motives specific to this tradition, ilustrated by many authors of Spanish Golden Age, which she declines in the argument of her play and which is enriched by the descriptions from the inquisitorial trials. From the initial invocation of the infernal powers, to the love spells or even animal metamorphosis or extraordinary displacements, Aldora coincides with the type of witch expected by the theatre audience. However, Ana Caro amplifies its scope by giving her character the power to resolve the dramatic conflict. This happy outcome highlights the playwright's desire to impose the supremacy of a trio of undefeated female characters formed by Aldora and the two powerful ladies, Queens Rosaura and Lisbella. Moreover, the structural homology between the character of the magician and the one of the author, emphasized by frequent funny meta-theatrical allusions in the dialogue, is symbolically carrying a subversive intention that illuminates the claims of the playwright and her quest of recognition.

\section{INDEX}

Mots-clés : magicienne, femmes, pouvoir, autrice, méta-théâtre

Palabras claves : maga, mujeres, poder, autora, metateatro

Keywords : magician, women, power, female author, meta-theatre

\section{AUTEUR}

\section{ISABELLE ROUANE SOUPAULT}

Centre aixois d'études romanes, Aix Marseille Université 